\title{
Further developments in binocular summation
}

\author{
RANDOLPH BLAKE and MICHAEL SLOANE \\ Cresap Neuroscience Laboratory, Northwestern University, Evanston, Illinois 60201 \\ and \\ ROBERT FOX \\ Vanderbilt University, Nashville, Tennessee 37240
}

\begin{abstract}
This paper reviews experiments that bear on the issue of binocular summation, the superiority of binocular over monocular viewing on various visual tasks covering studies published since the appearance of a previous review of this literature by Blake and Fox (1973). The experiments are grouped into three main categories-those that deal with the specificity of binocular summation (i.e., the extent to which inputs to the two eyes must coincide spatially and temporally), those that study binocular summation on suprathreshold tasks, and those that correlate binocular summation with other aspects of binocular function. The last section of the paper critically reviews several models of binocular summation.
\end{abstract}

In a 1973 review of studies on binocular summation, the superiority of binocular over monocular viewing on visual threshold tasks, Blake and Fox concluded that (1) binocular summation occurs for many tasks, including increment detection, form recognition, contrast sensitivity, and flicker detection, and (2) this enhancement in binocular performance stems from genuine neural interaction between the eyes, not just probability summation. The present review examines experiments on binocular summation that have appeared since the 1973 survey, and, as before, attention is limited to psychophysical experiments comparing monocular and binocular visual performance, excluding investigations of binocular rivalry and stereopsis. The review examines (1) the specificity of binocular summation, that is, the extent to which inputs to the two eyes must be matched along some dimension in order to yield binocular summation, (2) the evidence for binocular summation in visual performance with suprathreshold stimulation, (3) individual differences in the magnitude of binocular summation, and (4) recent theoretical accounts of binocular summation.

It should be noted that an improvement in binocular performance relative to monocular performance does not necessarily imply neural interaction between the two eyes, since statistical considerations alone dictate such an improvement: binocular viewing affords an observer two opportunities to detect a weak

We are grateful to Geoff Iverson, Joseph Lappin, James Hoffman, and Martha Teghtsoonian for their comments on an earlier version of this review. During preparation of this paper, R.B. was supported by a Career Development Award from NIH (EY00106). Requests for reprints should be sent to Randolph Blake, Cresap Neuroscience Laboratory, Northwestern University, Evanston, Illinois 60201. signal. This statistical property has been formalized as the probability summation hypothesis, and Blake and Fox (1973) discuss the various formulations of this hypothesis in detail. Recent work has generally recognized and sought to assess the potential contribution of probability summation, using either normative models (e.g., the integration model of signal detection theory) or empirical control conditions (e.g., temporal separation of the two monocular inputs). Moreover, very recently the logic of the probability summation hypothesis has been extended to tasks such as reaction time for suprathreshold stimuli (Blake, Martens, Garrett, \& Westendorf, 1980), and several models have been developed for treating the role of probability summation in these tasks. As a result, these recent studies have specified in more detail the particular conditions that promote genuine neural interaction between the eyes.

\section{STIMULUS SPECIFICITY}

The studies in this section deal with the extent to which the two monocular inputs must match along various stimulus dimensions in order to yield binocular summation.

\section{Retinal Correspondence}

In work on binocular summation, the monocular targets are usually arranged to stimulate corresponding areas of the two eyes. However, Thorn and Boynton (1974), using an increment detection threshold, studied the effects of stimulating noncorresponding areas. Binocular performance in the condition in which circular disks of light were delivered to retinal areas 4 deg disparate was poorer than that found with nondisparate stimulation. They concluded that, 
with noncorresponding stimulation, only probability summation was operative. Similarly, Westendorf and Fox (1977) measured increment detection thresholds for small flashes at several different degrees of disparity. Binocular summation in excess of probability summation occurred only at disparities which did not exceed a value producing diplopia during steady viewing. Within the fusional range, the sign of the disparity (crossed vs. uncrossed) had no influence on the magnitude of binocular summation.

Similar results have been reported for simple reaction time (RT) to a suprathreshold grating pattern (Blake, Martens, \& Di Gianfillipo, 1980; Harwerth, Smith, \& Levi, 1980). Binocular RT is faster than monocular RT whenever the test grating strikes corresponding retinal areas; outside the range of fusion, the advantage of binocular viewing is lost.

These studies show that stimulation of corresponding retinal areas is not crucial for binocular summation. Within some range, perhaps equivalent to Panum's area, disparate stimuli produce binocular summation in excess of probability summation. To date, there has been no thorough study of the magnitude of summation within the fusional range. Perhaps enhancement in binocular performance, while in excess of probability summation, varies with disparity. However, there are certain technical difficulties in studying this problem. Small, transient vergence errors associated with binocular viewing reduce the resolution for placement of disparate test targets; to minimize this problem, nonius markers are essential. Moreover, within central vision the fusional range itself is rather small, providing only a limited region for measuring any graded effects of disparity on binocular summation. However, thresholds could be measured in more peripheral portions of the visual field where fusional areas are larger. Also, the fusional range could be expanded by capitalizing on the fact that the size of Panum's area varies with contour sharpness (Kulikowski, 1978), disparity gradient (Burt \& Julesz, 1980), and target size (Kertesz, 1981). Finally, binocular summation for vertical and horizontal disparities could be compared to assess the unique contribution, if any, of stereoscopic mechanisms.

\section{Temporal Correspondence}

A close match of temporal as well as spatial characteristics is required if the two monocular inputs are to interact neurally and thereby yield improved binocular performance. If the interval between successively flashed monocular targets exceeds $100 \mathrm{msec}$, the two light flashes behave as independent events, with binocular performance falling to the level of probability summation (Matin, 1962). And, for successively presented grating targets, this upper limit may be as small as $50 \mathrm{msec}$ (Green \& Blake, 1981). In fact, asynchrony in excess of $100 \mathrm{msec}$ is now gen- erally regarded as the appropriate condition for specifying the level of performance expected from probability summation (Thorn \& Boynton, 1974; Westendorf \& Fox, 1974, 1975, 1977; Westendorf, Langston, Chambers, \& Allegretti, 1978). It is probably no coincidence that stereopsis, too, is impossible once the temporal interval between successive presentations of half-images exceeds $100 \mathrm{sec}$ (Ogle, 1963; Ross \& Hobgen, 1974). For asynchronies less than $100 \mathrm{msec}$, the magnitude of binocular neural summation varies inversely with the interval between successively presented monocular targets, with the maximum enhancement in binocular performance usually occurring with simultaneous presentation (Thorn \& Boynton, 1974). Interestingly, there is some evidence that for a given asynchrony the amount of binocular facilitation may depend on which eye is stimulated first (Thorn \& Boynton, 1974), suggesting a possible role of ocular dominance.

The two monocular targets need not be equivalent in duration. Westendorf, Blake, and Fox (1972) found that a brief, bright flash could be paired with a relatively long, dim flash without altering the magnitude of binocular summation, as long as the flash onsets coincided. Forced-choice detection rates for such a pairing were equivalent to those of flashes that were of equal duration and luminance, suggesting that the binocular detection system integrates energy in accordance with Bloch's law. This conclusion must be qualified by Westendorf and Fox's (1974) finding that equally detectable increments and decrements are not integrated binocularly but are treated as independent events. Pairing an increment in one eye with an equally detectable decrement in the other eye yielded detection rates around the level expected on the basis of probability summation, whereas pairs of increments or pairs of decrements yielded summation in excess of probability summation. Cohn and Lasley (1976) have confirmed and extended this finding. The absence of simple energy integration in the binocular detection system suggests that its presence in monocular detection (e.g., Ikeda, 1965) reflects the operation of peripheral, precortical mechanisms.

A few studies have examined the effects of temporal contrast modulation on binocular summation in grating detection. Over a range of spatial frequencies, Rose (1978) found almost a twofold enhancement in binocular sensitivity when gratings were flickered in counterphase at $3.5 \mathrm{~Hz}$, showing greater than linear summation in the motion detection system, since stationary, nonflickering gratings yielded a $40 \% \cong \sqrt{2}$ enhancement, which he assumed represented linear summation. The role of movement in binocular summation was directly studied by Arditi, Anderson, and Movshon (1981). They presented drifting gratings of the same spatial frequency separately to the two eyes, varying the directions of drift of the dichoptic gratings to be either the same or op- 
posite. At low spatial frequencies, gratings drifting in the same direction for the two eyes yielded a twofold enhancement in contrast sensitivity relative to monocular testing, an improvement greater than that expected on the basis of probability summation. With opposite directions of drift, binocular performance was no better than monocular, even under conditions of forced-choice testing. This complete absence of summation, including probability, suggested the possible involvement of interocular inhibition produced by this form of dichoptic stimulation. At higher spatial frequencies, however, binocular performance with the same direction of drift was only about $40 \%$ better than monocular, and, with opposite directions of drift, binocular performance achieved the level predicted on the basis of probability summation rather than falling to the monocular level. Considered together, these data indicate that at threshold the binocular combination of moving patterns is at least partially, and in some cases wholly, dependent on the two monocular components' sharing a common direction of motion.

Blake and Rush (1980) used a subthreshold summation technique to examine how closely the two monocular inputs must match in temporal frequency to yield facilitation in binocular grating detection. One eye received a grating that was barely below its own visibility threshold, while the other received a grating whose contrast was adjusted to threshold visibility. These dichoptic gratings were identical in spatial frequency and orientation but could differ in temporal frequency or temporal waveform. At low spatial frequencies, binocular summation depended very strongly on the similarity in flicker rates: Binocular summation was maximum when the temporal frequencies were identical for the two eyes and fell to half-strength when the two frequencies differed by only .3 octaves. At high spatial frequencies, there was little selectivity for temporal frequency; binocular summation occurred over a 1.5-octave range of disparities in temporal frequency. Blake and Rush also measured binocular sensitivity for gratings which flickered on-and-off sinusoidally either in phase (i.e., grating onset and offset occur in synchrony between the two eyes) or $180 \mathrm{deg}$ out of phase (i.e., grating onset in one eye was accompanied by grating offset in the other). At low spatial frequencies, gratings slowly flickering out of phase showed no binocular summation, whereas, at high spatial frequencies, out-of-phase and in-phase flicker yielded equivalent levels of binocular summation. Blake and Rush concluded that binocular grating detection must be served by at least two mechanisms distinguishable by their capacity to integrate monocular inputs over time and by their selectivity for temporal frequency.

Cavonious (1970) studied the effects of temporal phase using full-field, sinusoidal flicker, a display which could be characterized as having a very low spatial frequency (it is nonzero owing to the borders). At various flicker rates, the modulation depth of flicker was adjusted to threshold visibility. In-phase flicker yielded considerably lower binocular thresholds than did out-of-phase stimulation, and this difference between the two phase conditions was most pronounced at lower flicker rates. Sherrington (1906) found only modest differences between in-phase and out-of-phase stimulation, but he worked at the highest detectable temporal frequency, at which Cavonious found the smallest binocular phase effects.

\section{Spatial Frequency and Orientation Selectivity}

Several recent studies have asked how close in spatial frequency the two monocular inputs must be to yield binocular summation. The point of departure for these studies has been the observation by Campbell and Green (1965) that binocular contrast sensitivity for grating detection is higher than monocular sensitivity by the factor $\sqrt{2}$. This finding has since been confirmed (Arditi et al., 1981; Blake \& Cormack, 1979; Blake \& Levinson, 1977; Blake \& Rush, 1980; Blakemore \& Hague, 1972; Lema \& Blake, 1977; Levi, Harwerth, \& Smith, 1979; Rose, 1978), although some studies have noted that the magnitude of summation declines with increasing spatial frequency. Campbell and Green always presented identical spatial frequencies to the two eyes. Using the subthreshold summation technique, Blake and Levinson (1977) measured contrast thresholds for a test grating of fixed spatial frequency viewed by one eye while the other eye received a background grating of variable spatial frequency. Binocular sensitivity was greatest when the two eyes received identical spatial frequencies but fell steadily to the monocular level as the spatial frequency difference approached $1 / 2$ octave. This spatial selectivity of binocular summation was also found by Bacon (1976), who measured forced-choice detection performance under conditions of dichoptic grating stimulation. He also found that the two patterns must be in-phase spatially, so that the peaks in the spatial waveform fall on corresponding retinal areas; when the monocular components differed in relative phase, binocular performance approached the level of probability summation only. Phase-specific summation has also been reported by Green and Blake (1981).

In addition to its selectivity for spatial frequency and phase, binocular summation also requires that the two eyes receive very nearly matched orientations. This was first reported by Westendorf and Fox (1975), who measured forced-choice detection of rectangular flashes. Summation in excess of probability was found when both eyes received flashes of the same orientation, but, when the two eyes received orthogonal orientations, binocular performance was 
at the level expected from probability summation. In a more systematic study of dichoptic orientation disparity, Blake and Levinson (1977) measured grating contrast thresholds and found that binocular sensitivity was maximum when the two eyes received identical orientations and fell rapidly to the monocular level when the orientations differed by more than $15 \mathrm{deg}$. It is interesting to note that at suprathreshold contrasts, 15 deg represents the maximum orientation disparity that can be tolerated before fusion gives way to binocular rivalry.

\section{SUPRATHRESHOLD TASKS}

\section{Binocular Brightness Summation}

In the literature on binocular summation, perhaps the most controversial issue has been brightness summation between the two eyes. There are some studies purporting to demonstrate that a binocularly viewed test appears brighter than a comparison test viewed monocularly (De Silva \& Bartley, 1930; Fry \& Bartley, 1933; Leibowitz \& Walker, 1956), but these findings have been challenged on the grounds that brightness judgments were contaminated by lateral interactions between test and comparison targets (Engel, 1967; Levelt, 1965). Moreover, the notion of brightness summation seems incompatible with the wellestablished Fechner's paradox, which implies some form of brightness averaging between the two eyes. Finally, the issue has been complicated by controversy concerning the appropriate metric for expressing the monocular components in a binocular brightness judgment. Some (e.g., Levelt, 1965) have favored luminance, others (e.g., Teller \& Galanter, 1967) have chosen brightness, while still others (e.g., de Weert \& Levelt, 1974) have opted for discriminal responses. Whichever metric is chosen, the nature of the interaction (averaging, summation, or both) between the monocular components remains to be determined.

With one exception, contemporary models of binocular brightness have focused on the averaging outcome and have minimized or ignored brightness summation. Levelt's (1965) luminance averaging model and Engel's $(1967,1969)$ autocorrelation model both treat binocular brightness as a weighted sum of monocular responses. Because these weighted summation models predict zero contribution when one eye's target is zero luminance, they necessarily predict that a monocularly viewed target will be equivalent in brightness to the same target viewed binocularly. The centroid model of de Weert and Levelt (1974) is a modified weighted summation model that contains a parameter designed to account for a small brightness summation effect. This parameter assumes that even in the absence of stimulation an eye contributes to binocular brightness, supposedly due to incomplete dark adaptation. Thus, the centroid model allows for a monocularly viewed target to appear slightly dimmer than the same target viewed binocularly, because the monocular target's brightness is reduced by this small contribution from the nontested eye. In effect, this "dark light" parameter extends the conditions for Fechner's paradox to the case of exclusive monocular stimulation, thereby preserving the generality of averaging.

A rather different type of brightness summation model has been proposed recently by Curtis and Rule (1978). This alternative, the vector summation model, was based on results from direct scaling and category rating experiments. Observers judged the brightness of flashed, dichoptic luminance pairs; to avoid spatial interactions between test and comparison targets, a single, fused target was presented for judgment. The brightness of dichoptic targets differing in luminance was consistently judged as less than the brightness associated with the more intense of the two monocular targets presented alone. This outcome represents Fechner's paradox. When the two eyes received approximately equal luminances, binocular brightness was greater than the brightness of either component alone, an outcome evidencing brightness summation. Moreover, the magnitude of this summation effect was considerably greater than that predicted by the centroid model of de Weert and Levelt (1974).

Curtis and Rule (1978) proposed a model that describes binocular brightness as the sum of two monocular vectors whose lengths are proportional to the respective monocular brightnesses. Their vector summation model allows for substantial binocular brightness summation as well as for Fechner's paradox. Because vector summation includes an interaction term whose weight is related to the angle separating the two component vectors, the length of the resulting binocular vector can assume a value less than the longer of the two monocular vectors or in excess of either individual component. When the angle separating the monocular vectors falls between 90 and $120 \mathrm{deg}$, summation and averaging can result. Angles less than $90 \mathrm{deg}$ yield only summation, and angles exceeding 120 deg yield only Fechner's paradox.

The data of Curtis and Rule provide good evidence for a genuine brightness summation effect between the two eyes, under conditions designed to rule out confounding effects of spatial interactions within a monocular field. Their model furnishes a simple geometrical description of binocular brightness, although it does not provide an interpretation of the angle separating the two monocular vectors. There is also no provision in the model for weighting of the two monocular vectors, which could therefore limit the model to conditions in which contour strength (cf. Levelt, 1965) is equivalent for the two eyes. 


\section{Contrast Summation}

Recently, several papers (Birch, 1979; Iverson, Movshon, \& Arditi, 1981; Legge \& Rubin, 1981) have studied apparent binocular contrast when the two eyes view sinusoidal gratings of different contrast. All three studies seem to agree that no single model adequately describes the resulting binocular contrast. Birch (1979) concludes that the combination rule changes from vector summation to simple linear addition as spatial frequency is lowered. Legge and Rubin (1981) find evidence for vector summation in some conditions, dependent on the contrast of a standard matching grating. However, they also find conditions yielding a weak analog to Fechner's paradox, in which the apparent contrast of a monocular grating is actually reduced by the addition of a low contrast grating to the other eye. Iverson et al. (1981), on the other hand, find no instance of Fechner's paradox and, rather, find evidence for power summation with an exponent ranging from 2 (i.e., the case of vector summation) to around 4 (i.e., a situation in which a single eye strongly dominates the binocular percept).

Each of the three studies employed different matching techniques, which may contribute to the lack of complete agreement. Still, these studies are unanimous in demonstrating that binocular contrast does not obey a simple averaging rule.

\section{Reaction Time}

Because RT varies inversely with stimulus intensity, it provides a useful index for scaling binocular summation at suprathreshold levels. Several recent studies have found that RT to the onset of small light flashes is faster for binocular viewing. This result obtains throughout the binocular portion of the visual field (Gilliland \& Haines, 1975), with white as well as monochromatic light (Haines, 1977), and over a wide range of luminances and flash durations (Ueno, 1977). None of these studies deal with the potential contribution of probability summation to binocular RT, thus limiting their theoretical contributions.

Two RT studies (Blake, Martens, \& Di Gianfillipo, 1980; Harwerth, Smith, \& Levi, 1980) have examined probability summation in binocular RT. Both measured simple RT to the onset of sinusoidal gratings of various spatial frequencies and contrasts. Blake et al. found that binocular stimulation consistently yielded shorter RTs regardless of grating contrast or spatial frequency. Expressed in milliseconds, binocular RT was about $10 \%$ faster, but, in terms of the contrast necessary to evoke some criterion, RT binocular viewing surpassed monocular viewing by more than $40 \%$. Harwerth et al. obtained generally similar results except that larger individual differences were found. Also, they found that horizontal gratings yielded less binocular summation than vertical gratings, contrary to the results of Blake et al.

In both of these studies, binocular RT was faster than that measured under two control conditions designed to estimate the improvement in RT based on probability summation. One condition involved binocular stimulation of noncorresponding retinal areas, and the other involved presentation of orthogonally oriented gratings to the two eyes. Blake et al. showed that performance on these control conditions closely matched that predicted from a stochastic latency model treating binocular $\mathrm{RT}$ as a race between inputs to two channels with uncorrelated sensitivities. Blake, Martens, Garrett, and Westendorf (1980) have spelled out the logic of this probability summation model of RT in some detail.

\section{Afterimages and Aftereffects}

Binocular summation of brightness and the superiority of binocular RT imply that binocular viewing can generate a larger neural response than can monocular viewing. It follows, then, that the residual effects of binocular stimulation might be more pronounced than those produced by monocular stimulation. Observations relevant to this issue come from studies of afterimages and aftereffects.

A monocularly induced afterimage, although originating within the retina itself, transfers interocularly, indicating that the information integral to the monocular afterimage proceeds to a binocular locus (Day, 1958). Thus, it is not surprising that a binocularly induced afterimage is more robust than one that is induced monocularly: Binocular afterimages appear sooner, last longer (e.g., Misiak \& Lozito, 1951), and are less prone to fragmentation (Forde, 1971; Owen \& Chalfant, 1970; Wade, 1972). Forde and MacKinnon (1975) have shown that the greater stability of binocular afterimages cannot be explained solely on the basis of independent fluctuations of the two monocular afterimages. Rather, the robustness of binocular afterimages points to a genuine neural summation effect.

There is disagreement concerning the difference monocular or binocular adaptation makes to visual aftereffects. Several earlier papers (Bonnet \& Pouthas, 1972; Pickersgill \& Jeeves, 1964) reported that the motion aftereffect decayed more slowly following binocular adaptation, but the differences between these two conditions were small. Lehmkuhle and Fox (Note 1) have recently studied this question more systematically. They tested a simple model of the motion aftereffect that assumes that aftereffect duration is monotonically related to the number of adapted neural elements within an ensemble consisting of monocular/left-eye, monocular/right-eye, and binocular neurons. (The relative contributions of these 
different groups of neurons to adaptation were estimated using various other adaptation conditions such as interocular transfer.) Lehmkuhle and Fox found, as the model predicted, that the motion aftereffect lasted longer following binocular adaptation; in fact, the actual duration of the binocular motion aftereffect was very close to the value predicted from other adaptation conditions. Similar results and conclusions were reported by Lehmkuhle and Fox (Note 2) for the tilt aftereffect.

Moulden (1974), on the other hand, found no difference between monocular and binocular adaptation for either the motion or the tilt aftereffects. Likewise, Wade (1976) failed to find such differences in the case of the motion aftereffect, and Wade and Wenderoth (1978) found that monocular and binocular adaptation yielded tilt aftereffects of equivalent magnitude. While there are procedural differences among these studies, none seem obviously related to the presence or absence of a summation effect. The controversy may stem, in part, from saturation effects in the magnitude of the aftereffect under study. As a rule, aftereffects can grow in strength only to some level beyond which further adaptation has no effect. If an aftereffect were at or near full strength, differences between monocular and binocular adaptation would be difficult to measure. In their study of the threshold elevation aftereffects, Blake, Overton, and Lema (1981) purposely set the contrast of an adaptation grating to a presaturation level and found that binocular adaptation yielded a larger elevation in contrast threshold than did monocular adaptation. It remains to be seen whether the controversy surrounding binocular adaptation and the aftereffects of tilt and motion may be resolved in this manner.

\section{Visuomotor Coordination}

In a departure from the typical research on binocular summation, Jones and Lee (1981) compared monocular and binocular performance on a battery of more "naturalistic" tasks designed to preclude any contribution from stereopsis. Those tasks included threading a needle, pouring water into a small container, and tracking an irregularly moving target. There were also some timed visual search tasks in which the targets were complex forms (e.g., a picture of an octopus in camouflage). On all these tasks, observers were faster and more accurate when using both eyes. It is noteworthy that observers were allowed unlimited viewing time and free head movements. The authors concluded that the most important role of binocular viewing is not to promote stereopsis but to facilitate visuomotor coordination and exteroception (or, in Gibsonian terms, active pick-up of visual information from the ambient array). Moreover, the authors believe this facilitation is due to the higher order registration of binocular concordance (i.e., matching of information in the two eyes), and they claim that this process is more than simple probability or neural summation. Jones and Lee leave the concept of binocular concordance largely unexplained; nevertheless, their experiments represent a desirable extension of the binocular summation question to settings that are ecologically more realistic.

\section{BINOCULAR SUMMATION AND STEREOPSIS}

As with other measures of visual function, there are large individual differences in the magnitude of binocular summation. This aspect of summation has been explicitly examined in the last few years, with special emphasis on the relation between summation and other aspects of binocular function. This work has been inspired partly by the physiological finding that discordant binocular input (e.g., induced strabismus) early in life can disrupt the normal pattern of binocular interaction among visual cortical neurons (e.g., Baker, Grigg, \& von Noorden, 1974).

Using a detection task involving very briefly presented, foveally viewed forms, Williams (1974) found evidence for binocular summation in 8 of 10 observers; the two observers showing no superiority of two-eye viewing were also unable to appreciate depth in conventional anaglyphic plates and random-element stereograms. Both these individuals who lacked stereopsis had small angle misalignment of the two eyes which, Williams conjectured, could produce some form of inhibition in which one eye suppressed any contribution from the other. Significantly, these two observers failed to show even probability summation, an outcome consistent with the interocular inhibition hypothesis.

Lema and Blake (1977) assessed binocular summation over a range of spatial frequencies in observers with good stereopsis as well as in nonamblyopic observers who lacked stereopsis. Binocular thresholds were $30 \%$ to $50 \%$ lower than monocular thresholds in normal observers but were essentially equivalent in three of four stereoblind observers. For the fourth stereoblind observer, binocular and monocular thresholds were equivalent at all but the highest spatial frequencies, at which binocular performance exceeded monocular by $30 \%$, suggesting that losses in binocularity may be specific to certain spatial frequencies. Three of the stereoblind observers had histories of childhood strabismus; the fourth had no history of eye misalignment or any other obvious impediment to normal binocular function. Lema and Blake attributed the absence of binocular summation in stereoblind observers to a paucity of binocularly innervated neurons. In this regard, it is interesting that cats with demonstrated losses in cortical binocularity also show no summation of contrast threshold when tested behaviorally, whereas cats with the normal 
proportions of binocular neurons do exhibit binocular summation (von Grunau, 1979); cats with few binocular neurons also lack stereopsis (Blake \& Hirsch, 1975).

The absence of binocular summation in stereoblind observers has been confirmed by Levi, Harwerth, and Smith (1979) and by Westendorf et al. (1978). The latter group, using a forced-choice detection task, found that stereoblind observers performed at the level predicted on the basis of probability summation and interpreted this as evidence that the two eyes behaved independently at threshold.

This pattern of results appears to hold for suprathreshold levels of stimulation. Blake, Martens, and Di Gianfillipo (1980) found that on a simple RT task stereoblind observers performed binocularly at the level of probability summation, whereas in normal observers binocular performance was in excess of probability. Levi, Harwerth, and Manny (1979) failed to find even probability summation in stereoblind, amblyopic observers. This discrepancy could be due to the fact that Blake et al. adjusted the contrast level for each eye separately to yield equivalent RTs under monocular testing, to compensate for the consistently slower RTs associated with the amblyopic eye. Levi et al. did not perform such a compensation, which means that in their study binocular RT was always determined by the more sensitive, nonamblyopic eye. A major difficulty in studying binocular summation in people with disorders in binocular vision is that they frequently possess a strongly dominant eye and may exhibit chronic suppression and/or strabismus. So, even though viewing may be nominally binocular, testing may be functionally monocular. To minimize these difficulties, great care must be taken to insure proper alignment of the eyes by using prisms or mirrors, and allowance must be made for differences in acuity when adjusting the exposure conditions (e.g., contrast) for the two eyes.

To summarize, individuals with deficient stereopsis show little, if any, binocular summation, suggesting that stereopsis and summation may be mediated by a common neural mechanism. But, even among observers with good stereopsis, there are marked individual differences in binocular summation (e.g., Westendorf et al., 1972). These individual differences seem unrelated to the keenness of stereoacuity, and they are found under conditions of forced-choice testing that would minimize nonsensory criterion effects. Conceivably, small idiosyncratic errors in vergence could cause stimulation of slightly noncorresponding retinal areas, leading to a reduction in binocular summation. Alternatively, these individual differences could reflect variations in the extent of binocular neural interaction unrelated to stereopsis. Without an explicit model of binocular neural summation, an account of individual differences in the degree of summation remains largely speculative.

\section{THEORETICAL CONSIDERATIONS}

Despite the wealth and variety of data, binocular summation has received limited theoretical treatment, except for the issue of neural vs. probability summation. This paper has reviewed studies showing that binocular performance typically exceeds that predicted on the basis of probability summation, a finding that implicates neural interaction as the basis for summation. Many recent papers on binocular summation have included in their discussions some appeal to the involvement of binocularly innervated, cortical neurons. Such an appeal merely restates the empirical finding of neural summation and does not furnish any new insights about the details of its occurrence. Moreover, without an explicit model relating threshold judgments to neural activity, it seems premature to develop theories of binocular summation based on specific response properties (e.g., firing rate) of binocular neurons. It is feasible, though, to develop models of summation specifying such general modes of interaction between the two eyes as linear summation, probability summation, and binocular inhibition, when these modes are operationally defined in terms of the difference between monocular and binocular performance.

This latter approach, while not involving detailed physiological assumptions, does require the selection of some measurement scale for performance. This, in turn, means that a particular model may be specific to that metric of performance. To illustrate, consider the probability summation hypothesis, which asserts that the two eyes behave as independent detectors. All the formulations developed for assessing probability summation utilize percent correct or $d^{\prime}$ for predicting binocular performance based on two independent chances to detect. How, then, does one apply such formulations in situations in which performance is expressed in terms of some minimum energic value (e.g., luminance) necessary for threshold visibility? To do so requires making assumptions about the relation between percent correct performance and that energic dimension (e.g., assumptions about the equivalence of slopes of the monocular and binocular psychometric functions). While such assumptions may be justifiable, this requirement underscores the fact that the generality of a model will depend on the degree to which binocular performance on different tasks can be described on a scale common to all tasks. This problem is especially difficult when one attempts to model outcomes on both threshold and suprathreshold tasks.

In view of these considerations, it is not surprising that few formal models of binocular summation have been developed. In the following sections, we shall describe those few neural models that have been proposed to account for binocular performance on threshold tasks. 


\section{Energy Summation}

According to this model, the binocular detection system responds to the sum of the activity delivered by the two monocular pathways, in much the same manner that a pair of inputs would be integrated within a single eye. In its simplest form, the model assumes that doubling the energy in one eye is equivalent to delivering half of that energy to each eye separately; a central, binocular integrator will receive the same total input in both cases.

Although not explicitly called the energy summation model, this line of reasoning was first elaborated by Sherrington (1906) in his classic work on critical flicker frequency (CFF). Sherrington reasoned that, if activity from the two eyes were summed additively, the experience of flicker would be abolished when a train of monocularly visible flashes was presented out of phase dichoptically so that the summed frequency exceeded the CFF. In effect, he was assuming that the light phase of a flash to one eye would fill in the dark phase of the flash to the other eye. Because this and similar predictions were not realized, Sherrington rejected the idea of energy summation in favor of an independence model that treated the two eyes as separate visual channels brought together only after complete monocular analysis.

Earlier experiments on binocular summation employed monocular targets equivalent in energy, a condition that cannot really test the energy summation model. Several more recent experiments, however, were explicitly designed to evaluate this model. Support for energy summation comes from the study (Westendorf et al., 1972) showing that monocular flashes of unequal luminance and duration were equivalent in detectability to a pair of identical monocular flashes. Under those conditions, the binocular detection system responded only to the total energy of each monocular flash; however, this could result if energy integration occurs at a point in the visual pathway prior to binocular combination. A more decisive test of energy summation is provided by comparing the detectability of dichoptic pairs consisting of a luminance increment and a luminance decrement. Under these conditions, the model fails. Rather than the proportional increase in threshold predicted by the model, the threshold for detection of a dichoptic pair of opposite contrast flashes is actually lower than threshold for either flash alone (Cohn \& Lasley, 1976; Westendorf \& Fox, 1974). Similarly, binocular summation between gratings flickering temporally out of phase between the two eyes (Blake \& Rush, 1980) constitutes evidence against the energy summation model.

\section{Dual Process Model}

Cohn and Lasley (1976) proposed that binocular detection is served by two separate mechanisms, one that computes the difference between the two monocular signals and one that adds them. These summing and subtractive processes, in turn, pool their outputs in the interests of detection. The results from their study using dichoptic combinations of increments and decrements provided the impetus for this model (which is reminiscent of Fry and Bartley's, 1933, summation/inhibition model). In a later set of experiments, Cohn, Leong, and Lasley (1980) showed that a dioptic (i.e., identical) pair of increments could be discriminated from a dichoptic pair consisting of an increment and a decrement, even when these pairs of stimuli were equally detectable. This outcome was interpreted as evidence for the existence of two separate binocular detection mechanisms.

Although intriguing, this two-process model needs further elaboration. Cohn and Lasley (1976) state that the outputs from these two mechanisms are weighted, but they do not specify the rules governing the assignment of weights, other than to assume likelihood ratio processing. (Presumably, the weighting will be some function of the luminance disparity between the two monocular signals.) Furthermore, the model is difficult to distinguish from the alternative stipulating that the two eyes behave as independent detectors (i.e., probability summation) when presented with dissimilar stimulation (Westendorf \& Fox, 1974). Both models make qualitatively similar predictions, and the quantitative difference between these predictions depends on the particular formulation used to derive estimates of probability summation. There are several a priori reasonable formulations of probability summation that lead to different predictions regarding binocular performance. Without a firm commitment to one of these models, it becomes arbitrary to compare the two-process model with probability summation. A more fruitful approach for distinguishing these two alternatives would be to repeat Cohn and Lasley's experiment using asynchronous presentation of the two dissimilar flashes, which provides an unambiguous, empirical estimate of the performance expected on the basis of probability summation. A drop in binocular performance relative to the synchronous presentation of dissimilar flashes would provide evidence in favor of the two-process model. Limited measurements by Westendorf and Fox (1974) suggest that such a drop in performance does not occur, but more thorough testing is needed to resolve this issue.

\section{Campbell and Green Model}

In their study of monocular and binocular grating detection, Campbell and Green (1965) noted that binocular sensitivity exceeded monocular by about $40 \%$, a value suggestively close to $\sqrt{2}$. They proposed the following model based on this result: "Our findings may be explained if it is assumed that when identical 
images are fed to two matched eyes their outputs are summed and that these outputs are noisy; that is, contain spurious extra components not correlated in each channel. Because the standard error of the sum of $\mathbf{n}$ independent measurements of a random or noisy process decreases as $\sqrt{\mathrm{n}}$, an observer using two eyes can obtain two measurements which thus permit a $\sqrt{2}$ lower contrast to be detected"' (p. 192). The last sentence suggests that their model could be construed as a probability summation model, in as much as binocular viewing is characterized as two independent samples from a random process. In fact, expressed in this fashion, their formulation closely resembles the integration model of signal detection theory (Green \& Swets, 1966), which specifies the performance expected from multiple independent observations. However, Campbell and Green explicitly disavow this kind of probabilistic mechanism, instead asserting that their model "requires the actual physical summation of signals from the two eyes" (p. 192).

Actually, this latter interpretation of their model (i.e., neural summation) can readily account for the $\sqrt{2}$ superiority of binocular viewing without involving multiple, random sampling. Because the monocular channels contain uncorrelated noise, the standard deviation of the binocular signal-plus-noise distribution will increase, relative to the monocular distribution, by $\sqrt{2}$, whereas the mean of the binocular distribution will double. On the assumption of likelihood ratio processing, this leads to the prediction that binocular thresholds will be lower than monocular by $\sqrt{2}$. This restatement of the decision rule in their model identifies it unambiguously as a neural summation model.

The Campbell and Green model would be appealing if for no other reason than the empirical ubiquity of the $\sqrt{2}$ summation factor. Blake and Fox (1973), in their earlier review, noted that $\sqrt{2}$ summation has been observed on a number of threshold tasks including increment detection, flicker sensitivity, and spatial resolution. Let us now consider several features of this model in more detail.

The assumption of uncorrelated noise in the two monocular channels is tantamount to assuming that false alarms (i.e., spurious noise events that exceed the criterion level) occur independently between the two eyes. This assumption, although difficult to test directly, seems warranted for several reasons. First, within a single eye, sensitivity is uncorrelated for different retinal areas at any given moment (Eriksen, 1966). It seems reasonable to generalize this finding to retinal areas in different eyes, especially since the putative noise in Campbell and Green's model arises prior to the level of binocular combination. A second relevant finding comes from the noise-masking experiment by Braccini, Gambardella, and Suetta (1980). They presented either correlated or uncorrelated visual masking noise separately to the two eyes and in both conditions measured binocular contrast thresholds. To the extent that noise introduced externally mimics the internal noise posited by Campbell and Green, one would expect correlated noise to yield greater masking than uncorrelated noise. This was, in fact, the result obtained, and the finding has since been confirmed (Anderson \& Movshon, 1981). Of course, this finding in itself does not constitute proof that internal monocular noise is uncorrelated, but it is strongly suggestive of that.

While the assumption of uncorrelated monocular noise seems reasonable, there is one complicating factor. To account for the $\sqrt{2}$ lowering of binocular thresholds, the model must assume that, during monocular testing, only noise from the tested eye affects detection, while during binocular testing, noise from both eyes is involved. In effect, the mechanism responsible for summing activity in the two eyes must somehow recognize when only one eye receives stimulation and then exclude the noise from the unstimulated channel. To accomplish this, an interocular suppression mechanism could be incorporated into the model. However, such a mechanism would have to operate regardless of whether the nontested eye views a uniform field or is simply occluded, since $\sqrt{2}$ summation is found under both conditions (Campbell \& Green, 1965). To avoid this problem of monocular suppression, the model could be modified by postponing the introduction of noise until after summation of the two monocular signals. Then, however, the model predicts an improvement in binocular performance by the factor of 2 , not $\sqrt{2}$, because the monocular signals sum completely with no accompanying increase in noise strength. This modification, while more simply solving the problem of noise from an untested eye, is unacceptable as long as the weight of the evidence favors the $\sqrt{2}$ prediction.

In summary, the model of Campbell and Green, although appealing because of its predictive validity, needs to be reformulated to make explicit the role of noise in monocular vs. binocular detection.

\section{FINAL COMMENTS}

In the last 7 years, there has been continued interest in the problem of binocular summation, resulting in new insights about the neural mechanisms underlying enhanced binocular performance. In particular, much has been learned about the specificity of binocular summation, and the issue of individual differences, especially as it relates to other aspects of binocular function, has received increasing attention. Also during this recent period, there has been an increased awareness of the potential contribution of probability summation to binocular performance. This awareness has occasioned the development of 
more refined procedures, both analytical and empirical, for deriving estimates of probability summation. (As an aside, it is interesting to note that a similar concern about the contributions of probability summation has emerged in the work on spatial channels in human vision-e.g., see Graham, Robson, \& Nachmias, 1978). Finally, since our previous review of this literature, methodological advances have served to expand the study of binocular summation to suprathreshold levels of stimulation. Given these advances, it may now be opportune to begin relating binocular summation to other phenomena of binocular vision such as interocular transfer, binocular rivalry, and stereopsis, and to consider integrating these various phenomena into a comprehensive theory of binocular vision.

\section{REFERENCE NOTES}

1. Lehmkuhle, S., \& Fox, R. Binocular interaction of the motion aftereffect: $A$ simple linear model. Paper presented at the Annual Meeting of the Association for Research in Vision and Ophthalmology, Sarasota, Florida, 1975.

2. Lehmkuhle, S., \& Fox, R. Viewing conditions determine tilt aftereffect magnitude. Paper presented at the Annual Meeting of the Midwestern Psychological Association, Chicago, 1976.

\section{REFERENCES}

Anderson, P. A., \& Movshon, J. A. Detection of binocular contrast combinations. Supplement to Investigative Ophthalmology and Visual Science, 1981, $20,47$.

Arditi, A. R., Anderson, P. A., \& Movshon, J. A. Monocular and binocular detection of moving sinusoidal gratings. Vision Research, 1981, 21, 329-336.

BAcon, J. H. The interaction of dichoptically presented spatial gratings. Vision Research, 1976, 16, 337-344.

Baker, F. H., Griga, P., \& von Noorden, G. K. Effects of visual deprivation and strabismus on the response of neurones in the visual cortex of the monkey, including studies on the striate and prestriate cortex in the normal animal. Brain Research, $1974,66,185$.

Birch, E. E. Binocular processing of contrast: A vector sum model. Supplement to Investigative Ophthalmology and Visual Science, 1979, 18, 246.

Blake, R., \& CoRmack, R. H. On utrocular discrimination. Perception \& Psychophysics, 1979, 26, 53-68.

Blake, R., \& Fox, R. The psychophysical inquiry into binocular summation. Perception \& Psychophysics, 1973, 14, 161-185.

Blake, R., \& Hirsch, H. V. B. Deficits in binocular depth perception in cats after alternating monocular deprivation. Science, 1975, 190, 1114-1116.

Blake, R., \& Levinson, E. Spatial properties of binocular neurones in the human visual system. Experimental Brain Research, 1977, 27, 221-232.

Blake, R., Martens, W., \& Di Gianfillipo, A. Reaction time as a measure of binocular interaction in human vision. Investigative Ophthalmology and Visual Science, 1980, 19, 930-941.

Blake, R., Martens, W., Garrett, A., \& Westendorf, D. Estimating probability summation for binocular reaction time data. Perception \& Psychophysics, 1980, 27, 375-378.

Blake, R., Overton, R., \& Lema, S. A. Interocular transfer of visual aftereffects. Journal of Experimental Psychology, 1981, 7, 367-381.

Blake, R., \& Rush, C. Temporal properties of binocular mech- anisms in the human visual system. Experimental Brain Research, $1980,38,333-340$.

Blakemore, C., \& Hague, B. Evidence for disparity detecting neurones in the human visual system. Journal of Physiology (London), 1972, 255, 437-455.

Bonnet, C., \& Pouthas, V. Apparent size and duration of a movement aftereffect. Quarterly Journal of Experimental Psychology, 1972, 24, 275-281.

Braccini, C., Gamberdella, G., \& Suetta, G. A noise masking experiment in grating perception at threshold: The implications on binocular summation. Vision Research, 1980, 20, 373-376.

Burt, P., \& Julesz, B. A disparity gradient limit for binocular fusion. Science, 1980, 208, 615-617.

Campbell, F. W., \& Green, D. G. Monocular versus binocular visual acuity. Nature, 1965, 208, 191-192.

CAvonious, C. R. Binocular interactions in flicker. Quarterly Journal of Experimental Psychology, 1979, 31, 273-280.

Cohn, T. E., \& Lasley, D. J. Binocular vision: Two possible central interactions between signals from the two eyes. Science, 1976, 192, 561-562.

Cohn, T. E., Leong, H., \& LAsLey, D. J. Mechanisms for combination of binocular signals. Supplement to Investigative Ophthalmology and Visual Science, 1980, 18, 107.

Curtis, D. W., \& Rule, S. J. Binocular processing of brightness information: A vector-sum model. Journal of Experimental Psychology, 1978, 4, 132-143.

DAY, R. H. On interocular transfer and the central origin of visual after-effects. American Journal of Psychology, 1958, 71, 784-790.

De Silva, H. R., \& Bartley, S. H. Summation and subtraction of brightness in binocular perception. British Journal of Psychology, 1930, 20, 242-252.

De Weert, M. M., \& Levelt, W. J. M. Binocular brightness combinations: Additive and nonadditive aspects. Perception \& Psychophysics, 1974, 15, 551-562.

ENGEL, G. R. The visual process underlying binocular brightness summation. Vision Research, 1967, 7, 753-767.

ENGEL, G. R. The auto-correlation function and binocular brightness mixing. Vision Research, 1969, 9, 1111-1130.

ERIKSEN, C. W. Independence of successive inputs and uncorrelated error in visual form perception. Journal of Experimental Psychology, 1966, 76, 331-336.

Forde, G. Binocular after-images. Unpublished PhD thesis, University of Waterloo, 1971.

Fonde, J., \& Mackinnion, G. D. Binocular stimulation and the fragmentation of after-images. Quarterly Journal of Experimental Psychology, 1975, 27, 565-577.

Fry, G. A., \& BARTLEY, S. H. The brilliance of an object seen binocularly. American Journal of Ophthalmology, 1933, 16, 687-693.

Gilliland, K., \& Haines, R. F. Binocular summation and peripheral vision response time. American Journal of Optometry and Physiological Optics, 1975, 52, 834-839.

Graham, N., Robson, J. G., \& Nachmias, J. Grating summation in fovea and periphery. Vision Research, 1978, 18, 815-825.

Gheen, D. M., \& Swets, J. A. Signal detection theory and psychophysics. New York: Wiley, 1966.

Green, M., \& Blake, R. Phase effects in monoptic and dichoptic temporal integration: Flicker and motion detection. Vision Research, 1981, 21, 365-372.

Haines, R. F. Visual response time to colored stimuli in peripheral retina: Evidence for binocular summation. American Journal of Optometry and Physiological Optics, 1977, 54, 387-398.

Harwerth, R. S., Smith, E. L., \& Levi, D. M. Suprathreshold binocular interactions for grating patterns. Perception \& Psychophysics, 1980, 27, 43-50.

IKEDA, M. Temporal summation of positive and negative flashes in the visual system. Journal of the Optical Society of America, $1965,55,1527-1534$. 
Iverson, G. J., Movshon, J. A., \& Arditi, A. Binocular additivity of monocular contrasts. Supplement to Investigative Ophthalmology and Visual Science, 1981, 20, 224.

Jones, R. K., \& LEE, D. N. Why two eyes are better than one: The two views of binocular vision. Journal of Experimental Psychology: Human Perception and Psychophysics, 1981, 7, 30-40.

Kertesz, A. E. Effects of stimulus size on fusion and vergence. Journal of the Optical Society of America, 1981, 71, 289-293.

Kulikowski, J. J. Limit of single vision in stereopsis depends on contour sharpness. Nature, 1978, 275, 126-127.

LEGGE, G. E., \& RuBiN, G. S. Binocular interaction in suprathreshold contrast interactions. Perception \& Psychophysics, 1981, 30, 49-61.

LehmкUhle, S. W., \& Fox, R. On measuring interocular transfer. Vision Research, 1976, 16, 428-430.

LE IBOWITZ, H., \& WALKER, L. Effect of field size and luminance on the binocular summation of suprathreshold stimuli. Journal of the Optical Society of America, 1956, 46, 171-172.

Lema, S. A., \& Blake, R. Binocular summation in normal and stereoblind humans. Vision Research, 1977, 17, 69.

Levelt, W. J. M. On binocular rivalry (2nd ed.). The Hague: Mouton, 1965.

Levi, D. M., HARWe RTh, R. S., \& Manny, R. E. Suprathreshold spatial frequency detection and binocular interaction in strabismic and anisometropic amblyopia. Investigative Ophthalmology and Visual Science, 1979, 18, 716-725.

Levi, D., Harwerth, R., \& Smith, E. L. Humans deprived of normal binocular vision have binocular interactions tuned to size and orientation. Science, 1979, 206, 852-853.

Matin, L. Binocular summation at the absolute threshold for peripheral vision. Journal of the Optical Society of America, $1962,52,1276-1286$.

Misiak, H., \& LozitTo, C. C. Latency and duration of monocular and binocular afterimages. Journal of Experimental Psychology, 1951, 51, 247-249.

Moulden, B. After-effects, or a treatise of the consequences of adaptation to movement and tilt. Unpublished $\mathrm{PhD}$ thesis, University of Reading, 1974.

OGLE, K. N. Stereoscopic depth perception and exposure delays between images in the two eyes. Journal of the Optical Society of America, 1963, 53, 1296-1304.

Owen, D. H., \& Chalfant, C. Central integration effects with stabilized afterimages. Perception \& Psychophysics, 1970, 7, 284-286.
Pickergall, M. J., \& Cheeves, M. A. The origin of the motion aftereffect. Quarterly Journal of Experimental Psychology, $1964,16,103$.

Rose, D. Monocular vs binocular contrast thresholds for movement and pattern. Perception, 1978, 7, 195-200.

Ross, J., \& Hogben, J. H. Short-term memory in stereopsis. Vision Research, 1974, 14, 1195-1201.

She Rrington, C. S. The integrative action of the nervous system. New Haven: Yale University Press, 1906. (Republished 1947, 1961.)

Teller, D. Y., \& Galanter, E. Brightness, luminances, and Fechner's paradox. Perception \& Psychophysics, 1967, 2, 297. 300.

Thorn, F., \& Boynton, R. M. Human binocular summation at absolute threshold. Vision Research, 1974, 14, 445.

UENo, T. Reaction time as a measure of temporal summation at suprathreshold levels. Vision Research, 1977, 17, 227.

von Grunau, M. Binocular summation and the binocularity of cat visual cortex. Vision Research, 1979, 19, 813-816.

Wade, N. J. Orientation effects on line afterimages. Perception \& Psychophysics, 1972, 12, 409-416.

WADE, $\mathbf{N}$. J. On interocular transfer of the movement aftereffect in individuals with and without normal binocular vision. Perception, 1976, 5, 113-118.

WADE, N. J., \& WENDERoth, P. The influence of colour and contour rivalry on the magnitude of the tilt after-effect. Vision Research, 1978, 18, 827-835.

Westendorf, D., Blake, R., \& Fox, R. Binocular summation of equal energy flashes of unequal duration. Perception \& Psychophysics, 1972, 12, 445-448.

WestendorF, D., \& Fox, R. Binocular detection of positive and negative flashes. Perception \& Psychophysics, 1974, 15, 61-65.

Westendorf, D., \& Fox, R. Binocular detection of vertical and horizontal line segments. Vision Research, 1975, 15, 471-476.

Westendorf, D., \& Fox, R. Binocular detection of disparate light flashes. Vision Research, 1977, 17, 697-702.

Westendorf, D., Langston, A., Chambers, D., \& Allegretti, D. Binocular detection by normal and stereoblind observers. Perception \& Psychophysics, 1978, 24, 209-213.

Williams, $R$. The effect of strabismus on dichoptic summation of form information. Vision Research, 1974, 14, 307-309.

(Manuscript received December 5, 1980; accepted for publication June 15,1981 .) 